\title{
Model Reference Based Sliding Mode Controller with Nonlinear Plant
}

\author{
Dr. N. K. Yadav ${ }^{1^{*}}$, Dr. R. K. Singh ${ }^{2 \#}$ \\ ${ }^{1 *}$ Assistant Professor, Department of Electrical and Computer Engineering, \\ Arba Minch Institute of Technology, Arba Minch University, Ethiopia \\ 1nyadav24@gmail.com \\ 2\# Prof. Electrical Engineering Department, Motilal Nehru National Institute of Technology, \\ Allahabad, (UP - 211 004, India
}

\begin{abstract}
This paper designed for new algorithm for model reference system with nonlinear plant. The designed sliding mode controller is reduced the problem of unstructured systems, which are structuring the performance beyond unacceptable region. The plant being transformed and error dynamics created by incorporation of reference model. The error states are brought on the designed hyper plane for generating the desired performance of the system along with uncertainties. The designed algorithm is robust and works satisfactorily even when parametric variations happened. The designed algorithm will be useful for obtaining the desired performance of the nonlinear systems.
\end{abstract}

Keywords: Reference model, nonlinear plant, sliding mode controller, Linear matrix inequality

\section{INTRODUCTION}

Real world systems include nonlinearities or/and uncertainties. To obtain the desired performances, using the classical theories, are limited to find the solution of the problem up to not higher order systems. Sliding mode control (SMC) is a consisting of important properties of accuracy, robustness, and comfortable tuning and implementation. SMC technique to drive the system states onto a particular imaginary surface in the state space, which is nomenclature as sliding manifold. Once the states reached on sliding manifold by the reaching control, sliding mode control keeps the states on the close neighbourhood of the sliding manifld. The sliding mode control works on two phases. The first phase describes the sliding manifold so that the sliding motion satisfies design specifications. The second phase is describes the choosing the control law that will make the sliding manifold attractive to the system state [1]-[2]. There are two important advantages of sliding mode control. Firstly, the dynamic behavior of the system may be tracked by the specific chosen sliding function. Secondly, the closed loop dynamic response leads to completely insensitive to some of uncertainties. This method explores to model parameter uncertainties, disturbance and non-linearity that are bounded. In practical aspect SMC allows for controlling nonlinear processes on occurrence to external disturbances and heavy model uncertainties.

The sliding mode control techniques that allows to reach the desired performances on availability of internal and external disturbances. In addition to the stability, the regulating characteristics must be solved independently of uncertainties. In the literature, numerals techniques have been designed in continuous as well in discrete-time to solve the regulating problems for nonlinear systems. In the past decades many researches combine classical techniques with intelligent one, such as sliding mode with fuzzy logic systems or sliding mode with fuzzy systems [3-4], to worthful from the advantages of the two control techniques. For robust control system design, SMC has been well established for a wide range of nonlinear systems in continuous-time systems. It has major specific features such as quickly responsive for better transient performance. The salient advantages include: (i) robustness against a large amount of perturbation or model uncertainties; and (ii) control of certain class of complex nonlinear systems that are otherwise difficult to achieve. In SMC states are bounded to a desired sub-manifold of the state space. In reference (Emelyanov, 1967, Utkin, 1977) had proposed this concept of achieving desired dynamics by changing the controller structure. A strong speed switching control is used to bring the system state trajectory forcively to move along a chosen manifold in state space, is called sliding manifold.

Sliding mode control (SMC) systems have been studied broadly and have received many applications [4] - [6]. The control laws that drive the system's states to reach on sliding manifold and remain on the sliding surface, we attain the sliding mode. Once in the sliding mode, the dynamic system exhibits such invariance properties as robustness to on internal parametric variations and the external disturbances. The dynamic system performance of a SMC system is confirmed by the sliding surfaces upon which the control structure is switched. The sliding manifold is linear hyperplane, and the SMC of the linear systems have been well studied. However, most physical systems are nonlinear and so complex that may not be possible easily modeled mathematically. On the oppositely the mathematical treatment for unstructured system is still a problem in the modem control theory. Recently numeral specific results have been described in literature on the SMC problems for discrete-time systems therein reference [7] - [10], [14] - [18] and [19]. . The paper has been formulated as below: 
Section II, analyse the problem statement and description, section III, illustrates about the model reference and creating the error dynamics. Section IV, used to calculate the states feedback gain by linear matrix inequality method and section V, describes for designing of sliding manifold. Section VI, reveals the simulation work to proof of stability and section VII, deals with conclusion of the designed methodology.

\section{PROBLEM STATEMENT}

The nonlinear plant has been considered as below:

$$
\begin{aligned}
& \dot{x}(t)=f(x(t), u(t), t)) \\
& \dot{x_{p}}(t)=A_{p} x_{p}(t)+B_{p} u_{p}(t)+\xi(t)
\end{aligned}
$$

where $x_{p}(t) \in \mathfrak{R}^{n}$ and $u_{p}(t) \in \mathfrak{R}^{m}$ are the plant dynamics and control input vector. $A_{p} \in \mathfrak{R}^{n \times n}$ and $B_{p} \in \mathfrak{R}^{m \times n}$ are the plant's state matrix and control vector respectively and they are with appropriate dimensions. The nonlinearity $\xi(t)$ has been incorporated in the plant input.

Assumption: Pair $(A, B)$ are controllable.

\section{REFERENCE MODEL}

The reference model can be chosen as follows

$$
\dot{x_{m}}(t)=A_{m} x_{m}(t)+B_{m} u_{m}(t)
$$

Where where $x_{m}(t) \in \mathfrak{R}^{n}$ and $u_{m}(t) \in \mathfrak{R}^{m}$ are the reference model's dynamics and control input vector. $A_{m} \in \mathfrak{R}^{n \times n}$ and $B_{m} \in \mathfrak{R}^{m \times n}$ are the reference model's state matrix and control vector respectively and they are with appropriate dimensions.

The error dynamics can be computed between the plant and reference model states.

$$
\begin{aligned}
& e(t)=x_{p}(t)-x_{m}(t) \\
& e(t)=\left[\begin{array}{ll}
e_{1}(t) & e_{2}(t)
\end{array}\right]^{T}
\end{aligned}
$$

\section{CACLCULATION OF OPTIMAL GAIN MATRIX}

The state feedback gain is calculated by minimizing cost function. The performance index can be calculated by linear quadratic regulator method as follows:

$$
\begin{aligned}
& J=\frac{1}{2} \int_{t_{0}}^{\infty}\left[x_{1}^{T}(t) Q_{1} x_{1}(t)+x_{2}^{T}(t) Q_{2} x_{2}(t)\right] d t \\
& R=1 \\
& K=\operatorname{lqg}(A, B, Q, R)
\end{aligned}
$$

where $Q \in \mathfrak{R}^{m \times m}$ is the positive definite symmetric matrix.

\section{SLIDING SURFACE DESIGN}

The design of sliding surface consists of two phases. The first phase is to stable sliding surface in the state space. The second phase is to design an appropriate control law so that the system's states trajectories reached to the designed stable sliding surface in a desired time and maintain on to the sliding surface for all future time. Sliding mode control theory states that, under ideal sliding mode, sliding mode conditions are as below:

$$
\begin{aligned}
& \sigma(t)=0 \\
& \dot{\sigma}(t) .=0
\end{aligned}
$$

The designing of sliding surface is as follows

$$
\sigma(t)=\psi^{T} e(t)=0
$$

where $\sigma(t)$ is a sliding surface 


$$
\begin{aligned}
& \sigma(t)=\psi^{T} e(t)=\left[\begin{array}{ll}
\psi_{1} & I
\end{array}\right] e(t) \\
& \psi_{1}=\left(A_{m}-B_{m}^{T} K\right) \\
& \sigma(t)=\left[\begin{array}{ll}
\psi_{1} & I
\end{array}\right]\left[e_{1}(t) \quad e_{2}(t)\right]^{T}=0
\end{aligned}
$$

where $e(t)=\left[\begin{array}{l}e_{1}(t) \\ e_{2}(t)\end{array}\right]$

$$
\begin{aligned}
& \sigma(t)=\psi_{1} e_{1}(t)+e_{2}(t)=0 \\
& \psi_{1} e_{1}(t)+e_{2}(t)=0 \\
& e_{2}(t)=-\psi_{1} e_{1}(t) \\
& \dot{e}(t)=A e(t)+B u(t)
\end{aligned}
$$

The tracking error of the system must be zero

$$
A e(t)+B u(t)=0
$$

The calculated control law has been generated as follows

$$
u(t)=-B^{-1} A e(t)
$$

Lemmas 1 [12]: Let any real vectors $a$ and $b$ and matrix $P>0$ of appropriate dimensions, the following inequality holds:

$$
a^{T} b+b^{T} a \leq a^{T} P a+b^{T} P b
$$

Lemmas 2 [13]: Let $Q=Q^{T}$ and $N$ and $H$ be real matrices of compatible dimension with $F$ satisfying $F^{T} F<I$, then $\left(Q+N F H+H^{T} F^{T} N^{T}<0\right)$ if and only if there exists a scalar function $\varepsilon>0$ such that $\left(Q+\varepsilon N N^{T}+\varepsilon^{-1} H^{T} H<0\right)$ or inequality

$$
\left[\begin{array}{ccc}
Q & \varepsilon N & H^{T} \\
* & -\varepsilon I & 0 \\
* & * & \varepsilon I
\end{array}\right]<0
$$

The above lemmas give a sufficient condition to guarantee the robust asymptotic stability of the sliding manifold dynamic.

\section{SIMULATION RESULTS}

\section{A. Stable sliding surface}

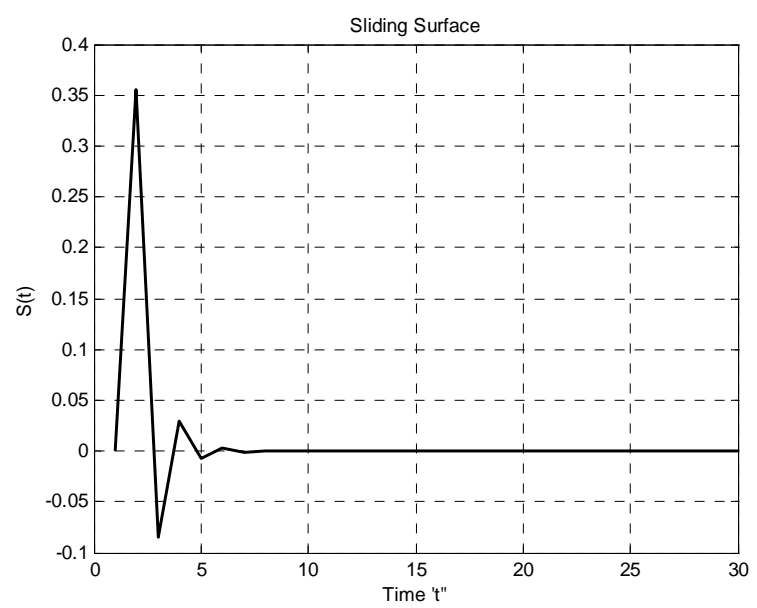

Fig. 1. Stable sliding surface 


\section{B. Stable system states}

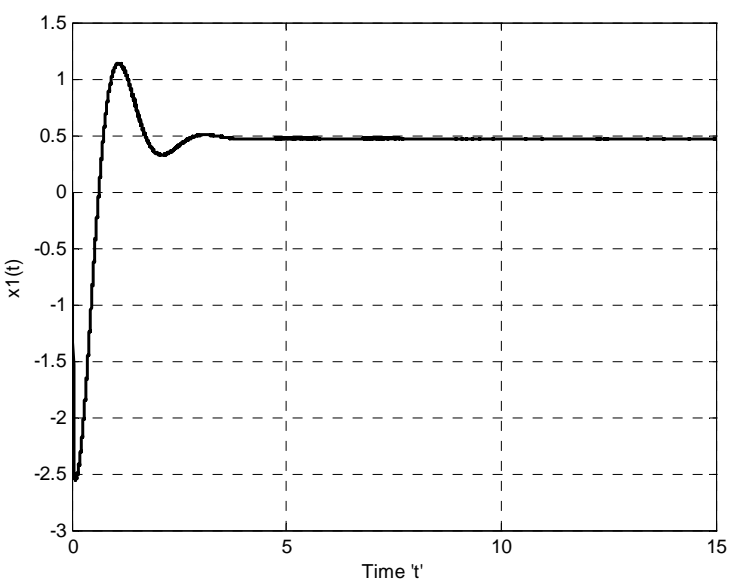

Fig. 2. Stable $x_{1}(t)$ state of the system

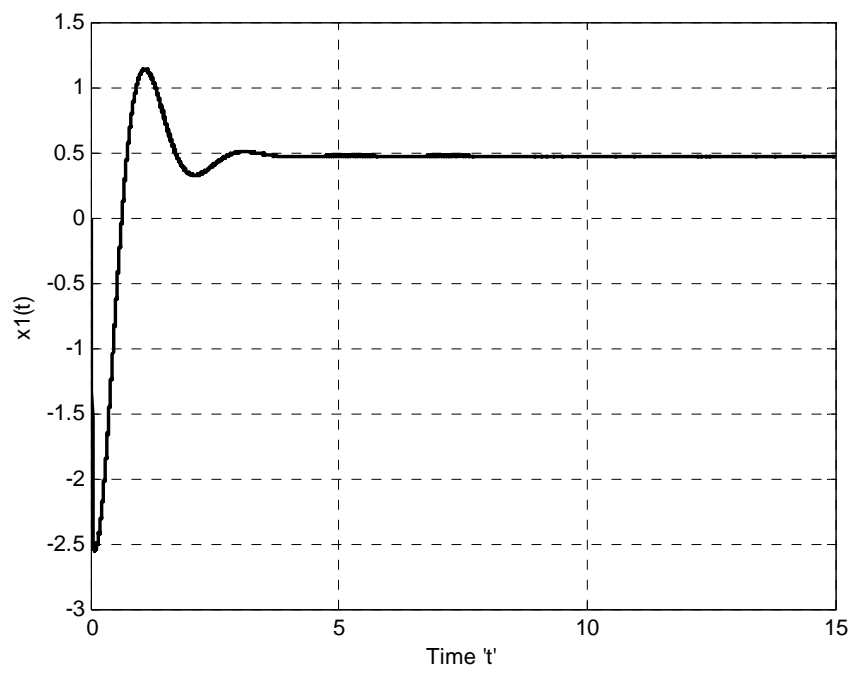

Fig.3 Stable $\mathrm{x}_{2}(\mathrm{t})$ state of the system

\section{VII.CONCLUSION}

The real systems are nonlinear in nature in the practical system and they are having ill defined model and the classical methods are quite cumbersome to generate the desirable stable performance by the system. algorithm that can be used for nonlinear. The designed algorithm will be more compatible for unstable systems. The proposed technique is more compatible even if in the variation of external condition or prevailing uncertainties. The problem simulation results reveal the stable desirable response of the nonlinear system. This technique will more suited to highly unstable system.

\section{REFERENCE}

[1] S. V. Emelyanov, (1967), "Variable structure control systems”, Moscow, Nauka..

[2] V. I. Utkin, (1977), "Variable structure systems with sliding modes”, IEEE Trans. on automatic Control, vol. 22, pp. 212-222.

[3] H. H. Choi, (1999), “An LMI approach to sliding mode control design for a class of uncertain time- delay systems”, In Proc. European Control Conference (Karlsruhe), pp. 1022-28.

[4] J. K. Hale and S. M. Luned Verduyn, "Introduction to functional differential equations”, Springer, New York, NY, 1993.

[5] D. Yue, Q. L. Han and C. Peng, "State feedback controller design of networked control systems”, IEEE Trans. Circuits System II Express brief, 2004, 51, (11), pp. 640-644.

[6] M. S. Mahmoud, "Robust control and filtering for time delay systems”, Marcel-Dekker, New York, NY, 2000.

[7] Jun Hu. Zidong Wang, Hui Gao and Lampros K. Stergioulas, "Robust sliding mode control for discrete stochastic systems with mixed time delays, randomly occurring uncertainties and randomly occurring nonlinearities”, IEEE Transaction on Industrial Electronics Vol. 59, no. 7, July, 2012, pp. 3008-3015.

[8] V. I. Utkin, Sliding modes in control optimization, Springer, Berlin, 1992.

[9] Jang Leeju, Xu Yangsheng, “A new method of switching surface design for multivariable structure systems”, IEEE Transactions on Automatic control, Vol. 39, N0.2, pp.414-149, 1994.

[10] K. Abidi, J. X. xu and N. H. Yu, “On discrete-time integral sliding mode control”, IEEE Trans. Autom. Control, Vol. 52, No. 4, pp. 709-715, Apr, 2007. 
[11] W. B. Gao, Y. F. Yang and A Homaifa, "Discrete-time variable structure control systems”, IEEE Trans. Industrial Electronics, Vol. 42, No. 2, pp. 112-122, Apr, 1995.

[12] Y. Q. Xia, G. P. Liu, P Shi, J. Chen, D. Rees and J. Liang, "Sliding mode control of uncertain linear discrete-time systems with input delays”, IET Control Theory Application Vol. 1, No. 4, pp. 1169-1175, Apr, 2007.

[13] M. Yan and Y. Shi, "robust discrete-time sliding mode control for uncertain systems with time varying state delay", IET Control Theory Application Vol. 2, No. 8, pp. 662- 674, Aug, 2008.

[14] A. J. Koshkouei, A. S. I. Zinober, "Sliding mode time delay systems". Proc. IEEE Workshop on variable structure control, Tokyo, Japan, 1996, pp. 97-101

[15] Y. Xia, Y. Jia, "Robust sliding-mode control for uncertain time-delay systems: an LMI approach', IEEE Trans. Autom. Control, 2003, 48, (6), pp. 1086-1092.

[16] Y. Zheng, G. M. Dimirovski, Y. Jing and M. Yang, “'Discrete-time sliding mode control of nonlinear systems”, Proc. 2007 American Control Conf., New York, USA, 2007, pp. 3825-3830.

[17] Furuta K., "Sliding mode control of a discrete systems" Systems and Control Letters Vol. 14, no. 2, pp.145-152,1990.

[18] Bandhyopadhyay B. and Deepak F., "High performance tracking controller for discrete plant using nonlinear sliding surface” IEEE transactions on Industrial Electronics, Vol.56, No.9, pp.3628- 3637, 2009.

[19] Linlin Li, Steven S Ding, Jianbin Qiu, Ying Yand and Yong Zhang "Weighted fuzzy observer-based fault detection approach for discrete-time nonlinear systems via piecewise-fuzzy Lyapunov Functions,” IEEE Transactions on Fuzzy System, vol. 24, no. 6, pp. 1320-1333, Dec. 2016.

\section{BIOGRAPHIES}

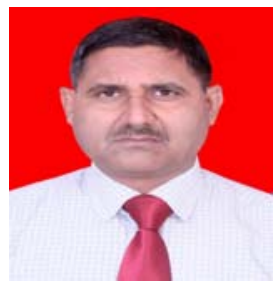

Dr. N. K. Yadav (S'09) is a faculty of Electrical and Computer Engineering department of Arba Minch Institute of Technology (AMIOT), Arba Minch University, Arba Minch, Ethiopia. He was graduated with degree in Electronics and Communication Engineering from Institution of Engineers (India), Calcutta, India in 1992, Master of Business Administration (M.B.A.) specialisation in Marketing management \& Personnel management from University of Pune, India 1997 and 1999 respectively, Master of Technology (M. Tech.) degree in Electrical Engineering with specialization in Control and Instrumentation and $\mathrm{Ph}$. D. in Control Engineering from Motilal Nehru National Institute of Technology (MNNIT), Allahabad (Uttar Pradesh) India, in 2003 and 2014 respectively. He is Chartered Engineered (India), elected Executive Committee member of Institution of Engineers (India), Faridabad, Local Centre and members of professional bodies LMISTE, IEEE, MIE, IEAE and IA. Eng. He is reviewer of national and international journals, /conferences He is having rich experiences of more than three decades in teaching, research and avionics industry as maintenance engineer in fighter aircrafts and associated systems. His current research interests are in areas of variable structure control, nonlinear system dynamics and control, robust control techniques, model reference adaptive control, sliding mode control and fuzzy / neural network modelling and control.

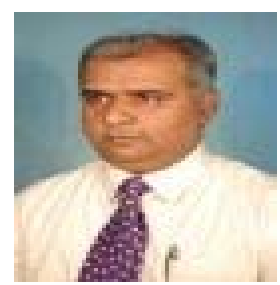

Dr. R. K Singh, is a Professor in the Department of Electrical Engineering, Motilal Nehru National Institute of Technology, Allahabad (MNNIT), India. He is having more than 26 years of teaching and research experience. His area of interest is dc-dc converters, electrical vehicle and distributed generation application. He is reviewer of national and international journal/conferences and working on research projects of government of India. Presently he is chairing IEEE local centre at Motilal Nehru National Institute of Technology, Allahabad. He is also a student's counsellor of IET local chapter of Motilal Nehru National Institute of Technology Allahabad (UP) India. . He is a Fellow of IE (India) and member of IEEE. 